Technical Report

\title{
Intrathecal Catheter-Syringe Adaptor for Short-Term Intrathecal Analgesia with an Externalized Pump: A Case Report
}

Denise Wilkes, MD, PhD, Michael Cook, MD, and Daneshvari Solanki, MD

From: University of Texas Medical Branch, Galveston, Texas.

Dr. Wilkes is with the Department of Anesthesiology, University of Texas Medical Branch, Galveston, TX. Dr. Cook is with the University of Texas Medical Branch, Galveston, TX. Dr. Solanki is Professor of Anesthesia and Pain Management, University of Texas Medical Branch, Galveston, TX.

Address correspondence: Denise WIlkes, MD, PhD

University of Texas Medical Branch Anesthesiology 301 University Blvd. Galveston, Texas 77555

E-mail: dwilkes@utmb.edu

Disclaimer: There was no external funding in the preparation of this manuscript.

Conflict of interest: None.

Manuscript received: 01/14/2010

Accepted for publication: 02/09/2010

Free full manuscript: www.painphysicianjournal.com
Background: In most patients, cancer pain is effectively treated with conservative medical management consisting of oral and/or transdermal analgesics. Cancer patients tend to fail conservative medical management near the end of their life expectancy, thus requiring alternative routes of analgesia such as intravenous, epidural, or intrathecal. The intrathecal route provides the most effective analgesia due to the close proximity of the opioid receptors in the spinal cord. Though there are many techniques that exist for intrathecal drug delivery, complications can limit effectiveness such as infection, bleeding, cerebrospinal fluid (CSF) leaks, post-dural puncture headaches (PDPH), pump and/or catheter malfunctions, or limitations of technical expertise. Therefore, an important goal in palliative cancer pain therapy is to use equipment that is going to have the fewest number of complications and will be the most familiar to the health care providers. We describe the combination of the Medtronic Indura 1P catheter, which has the least catheter-related complications and can be used with any external drug infusion pump. These are regular infusion pumps that the health care workers are familiar with so they can provide excellent and efficient service to the patient.

Methods: In an operating room, the intrathecal catheter was placed using sterile technique under fluoroscopic guidance. The epidural space was identified with loss of resistance technique. Then the introducer needle (supplied in the Indura 1P catheter kit) was advanced until free-flowing CSF was obtained. The spinal catheter was advanced into the intrathecal space through the introducer needle to lumbar 2-3 level. The catheter was tunneled subcutaneously $10 \mathrm{~cm}$ lateral to the catheter exit site. A syringe filling device was inserted into the catheter opening and was secured with silk suture. A luer lock syringe was attached to the syringe filling device and CSF was aspirated. The syringe filling device was capped and later attached to an external drug infusion pump.

Results: We report the successful use of the Medtronic Indura 1P, one piece intrathecal catheter, connected to the external drug pump for a 3 week period in a patient with metastatic cervical cancer for palliative pain control.

Limitations: Case report only.

Conclusion: This technique is simple to perform by pain specialists. The catheter modification allows the use of the Medtronic intrathecal catheter with standard external drug infusion pumps. This facilitates the patient's care in the hospice setting.

Key words: Intrathecal drug delivery, palliative medicine, intrathecal analgesia, intrathecal catheter complications, cancer pain, end of life pain control

Pain Physician 2010; 13:151-156 ancer patients in need of intrathecal palliative pain control often present to the pain specialist with less than 3 months to live. In most patients, cancer pain is effectively treated with conservative medical management consisting of oral and/or transdermal analgesics. Cancer patients tend to fail conservative medical management near the end of their life expectancy, thus requiring alternative 
routes of analgesia such as intravenous, epidural, or intrathecal. The intrathecal route provides the most effective analgesia due to the close proximity of the opioid receptors in the spinal cord. Though there are many techniques that exist for intrathecal drug delivery, the complications can limit the effectiveness such as infection, bleeding, CSF leaks, PDPH, pump and/or catheter malfunctions, or limitations of technical expertise can limit its effectiveness. Therefore, an important goal in palliative cancer pain therapy is to use equipment that is going to have the fewest number of complications and will be the most familiar to the health care providers. We describe the combination of the Medtronic Indura 1P catheter, which has the least catheter-related complications and the use of any external drug infusion pump. These are regular infusion pumps that the health care workers are familiar with so they can provide excellent and efficient service to the patient.

Studies have shown that implantable pumps are not cost effective in these patients with this short life expectancy (1). Therefore, the alternative approach is to tunnel the intrathecal catheter subcutaneously and use an external drug infusion pump (2-6). A recent metaanalysis of external intrathecal catheter complications shows complication rates were very low. Superficial infection, deep infection, bleeding, and neurologic damage were found to be $2.3,1.4,0.9$, and $0.4 \%$, respectively (7). They conclude that the use of these catheters is an effective and economical method of pain control (4). A CSF leak is a common risk after external intrathecal catheter placement (8). Although CSF leak is common, the rate of leak resolves with time and patients may or may not exhibit post-dural puncture headaches (PDPH) symptoms $(5,6)$, though PDPH is a common complication of intrathecal catheter placement. Burton et al (9) described a double catheter technique in order to facilitate the placement of a blood patch and to expedite the treatment of PDPH. Pain physicians will use epidural catheters for short-term intrathecal more often because of the availability, ease of use, lower cost, and presence of a catheter-syringe adaptor, which can be easily connected to external pumps. In the past we have used the Flextip Plus catheter (Arrow) in the intrathecal space, tunneled it subcutaneously, and connected it to a CADD Prizm external pump. In our experience, the epidural catheter produced CSF leaks, which was evident by the collection of CSF under the Tegaderm dressing. The CSF was observed within 24 hours but not all patients with CSF leaks were symptomatic. The CSF leak often caused alarm with the nursing and hospice staff, which required more attention and education by our pain specialists. Another disadvantage of this epidural catheter is that it has a single hole at the end and so is more prone to blockage and catheter failure.

We elected to adapt the Medtronic Indura P catheter for external drug pump use. The advantage of this catheter is that it is suitable for long-term use and contains a multi-orifice end. The disadvantage is that the connectors supplied with the Indura $\mathrm{P}$ catheter connect only to the SynchroMed® pump. This technical case report shows a modification of the Medtronic Indura 1P catheter, which will allow easy connectivity with standard external drug infusion pumps. In this report, we also show the other catheter-syringe adaptors that were tried and failed (Fig. 1).

\section{Methods}

A 63-year-old female presented to the oncologist with a 6-month history of severe abdominal pain and dysuria. She was diagnosed with stage IV squamous cell carcinoma of the cervix. She underwent chemotherapy and radiation therapy. The pain was poorly controlled with oral pain medications. Four months later, she was referred to the pain clinic. The pain was burning, sharp, and shooting and was located in the perineum. Neurolytic superior hypogastric and ganglion impar blocks were performed and oral medication was continued with morphine. A year later, the pain was located more diffusely over the abdomen, a 9-10/10 in severity, and was poorly controlled with oral medications. Over the next few months, the patient refused further interventions and opted for continued oral medical management. The patient became very debilitated. She could barely walk, eat, or drink and suffered from emesis and constipation. She was admitted to the hospital and diagnosed with gastric outlet obstruction secondary to compression by a mass. At this point, she desired palliative interventions. Her life expectancy was estimated to be 4-6 weeks. The goal for this hospitalization was to obtain pain control within 2-3 days with a regimen that could be maintained in a home based hospice setting.

The patient was informed of the risks and benefits of the procedure and she elected to proceed with the placement of the intrathecal catheter. This procedure was performed under a general anesthetic for patient comfort. A pre-operative dose of $1 \mathrm{~g}$ cefazolin was given intravenously. The patient was positioned on the operating room table in left lateral decubitus position with the knees pulled to the chest and the spine 


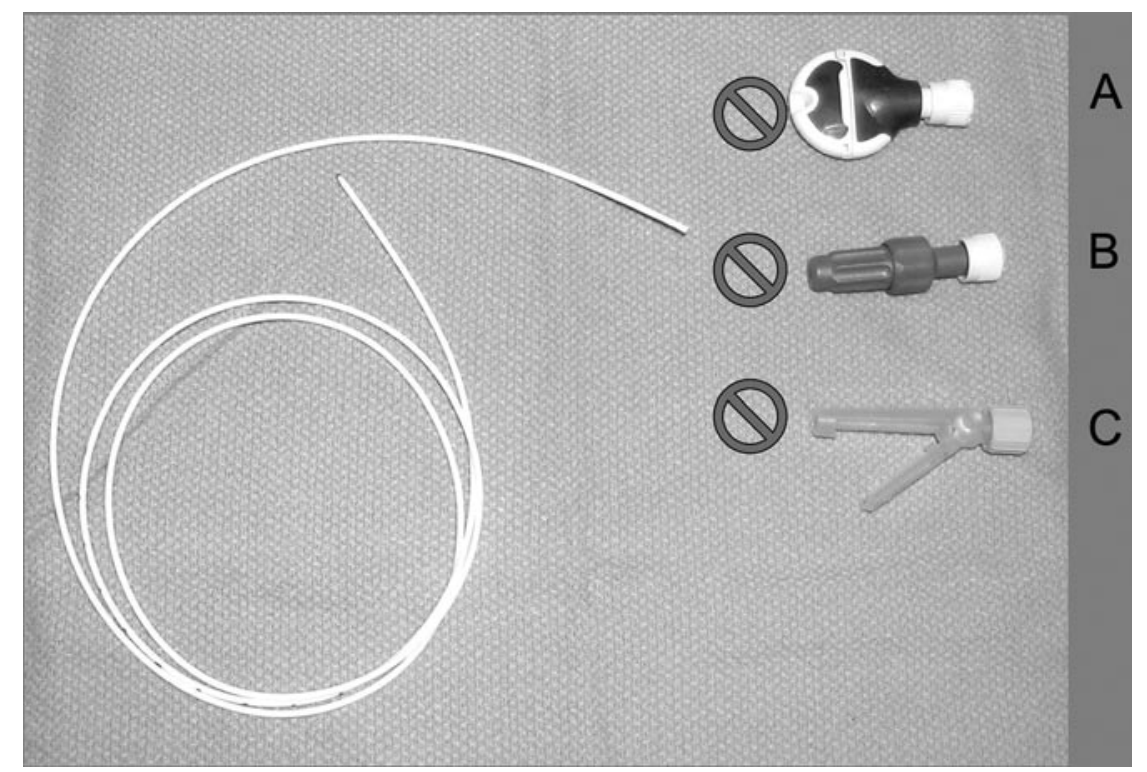

Fig. 1. Commercially available connectors not compatible with Medtronic Indura ${ }^{\circledR} 1 P$ catheter. A) Epimed catheter adaptor from the R.E.C. ${ }^{\mathrm{TM}}$ Radio-Opaque Epidural catheter. B) Snaplock adaptor from the StimuCath ${ }^{\mathrm{TM}}$ Continuous Nerve Block Kit. C) Clamp style catheter connector from the Contiplex Tuohy ${ }^{\circledR}$ Continuos Nerve Block Set.

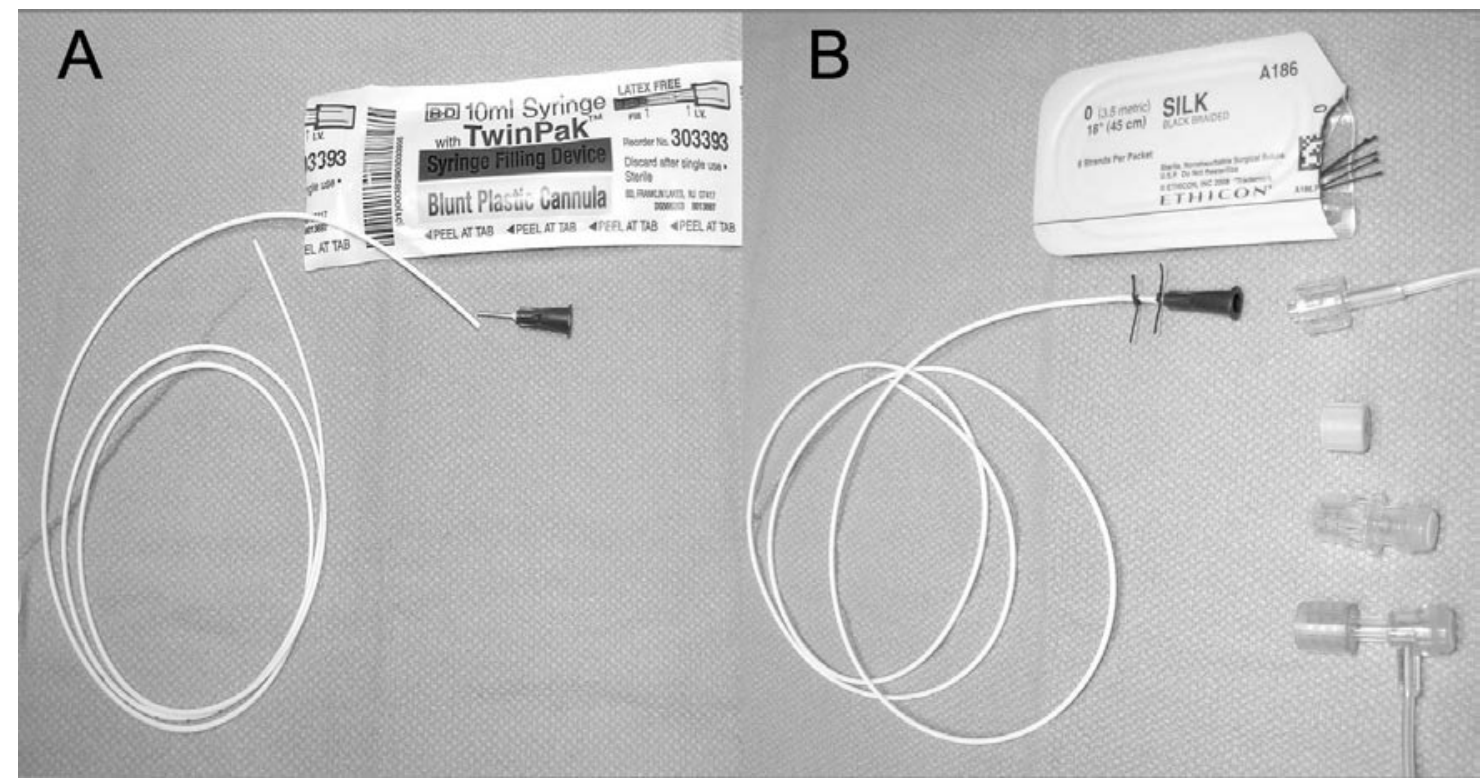

Fig. 2. A. A BD syringe with TwinPak. B. Syringe filling device inserted into the catheer opening and secured with 2-0 silk. 
flexed on the operating room table. The lumbar area was sterilely prepped and draped. Fluoroscopy was used to optimize the approach and to confirm intrathecal placement. A 15-gauge introducer needle, supplied in the Indura catheter kit (Medtronic 8709SC), was advanced under fluoroscopic guidance in a paramedian entry point between L4 and L5 interspace with the hub raised at approximately a $30^{\circ}$ angle. The epidural space was identified with loss of resistance technique. Then the introducer needle was advanced until the dura was penetrated and free-flowing CSF was noted. The spinal catheter was advanced into the intrathecal space and the catheter stylet was removed. In order to confirm placement, the syringe-filling device (BD syringe with Twin Pak ${ }^{T M}$ ) was inserted into the catheter distal end (Fig. 2A). A $3 \mathrm{~mL}$ syringe was attached to the syringe-filling device and CSF was aspirated. Then $1 \mathrm{~mL}$ of Omnipaque (iohexol, $300 \mathrm{mgl} / \mathrm{mL}$, GE Healthcare) was injected. The dye spread confirmed the position of the catheter in the intrathecal space. The syringe-filling device was then removed. A small incision was made at the catheter exit site. The introducer needle was removed over the catheter and re-inserted $10 \mathrm{~cm}$ lateral with the tip directed towards the catheter exit site. The catheter was then threaded through the introducer needle and the needle was removed. The catheter exit site incision was approximated with steri-strips and covered with a Tegaderm dressing. The catheter adjacent to the exit site was secured to the skin with a 2-0 silk and a V-winged anchor. The excess catheter was coiled and secured to the skin with a Tegaderm dressing. A syringe-filling device was inserted into the catheter opening and secured with 2-0 silk (Fig. 2B). A luer lock syringe was attached to the syringe-filling device and the position was confirmed with CSF aspiration. The syringe-filling device was capped and later attached to an external pump in the recovery area. The infusion consisted of morphine (Duramorph®) $20 \mathrm{mcg} / \mathrm{mL}$ and $50 \mathrm{mcg} / \mathrm{mL}$ bupivacaine at a rate of $1 \mathrm{~mL} / \mathrm{hr}$. The infusate bag was prepared under strict aseptic conditions in the pharmacy. The Infusate bag was changed every 2 days using aseptic technique at the bedside. We did not use a bacterial filter between the infusate bag and pump tubing since both were sterilely packaged and prepared and connected using aseptic technique. Another reason for the omission of the bacterial filter supplied with the Flextip Plus catheter kits is that the filter frequently caused the CADD (Continually Administrated Drug Delivery) Prizm pump to fail due to increased back pressure. The site of intrathecal catheter entry and tunneling site were examined for signs of infection such as erythema and/ or exudates. The pump tubing was changed after 11 days, when the patient was discharged home. The hospice company had one incidence of pump failure, which resolved with replacement of the pump. Otherwise, no complications were noted in the 3-week period. The intrathecal analgesia decreased the patient's pain score from 9-10/10 to 0-1/10 Visual Analog Score (VAS).

\section{Discussion}

This case report describes the method that provides an additional option to cancer patients with short life expectancies. Since this patient had failed oral therapy, the next step was to do an intrathecal morphine trial to provide better analgesia. Due to this patient's life expectancy being less than 3 months, she didn't qualify for a synchromed pump placement. Also the invasiveness of the surgery would have been very difficult for this patient to endure. Alternatives to an implanted intrathecal drug delivery system were either a tunneled catheter and external drug pump or an intrathecal catheter with a subcutaneous port. A tunneled catheter and external port was preferred over a subcutaneous port to avoid the possible dislodgement or disconnection from the port connection. This was a complication that we felt our hospice or pain practice could not remedy. Our standard method is to place a Flextip Plus catheter, tunnel the catheter subcutaneously, and connect to an external drug infusion pump. However, we were concerned that the Flextip Plus catheter might fail due to obstruction or dislodgement after a few weeks and would not last for the entire life expectancy of this patient. For this patient, who had been followed in the pain clinic for nearly 2 years, we wanted a system that had the least chance of failure.

Our plan was to modify the Medtronic Indura 1P catheter, which is designed for long-term use, so as to be able to connect to an external pump. We tried numerous connectors such as the Epimed catheter adaptor from the R.E.C. ${ }^{\text {TM }}$ Radio-Opaque Epidural catheter, Snaplock adaptor from the StimuCath ${ }^{\mathrm{TM}}$ Continuous Nerve Block Kit, and the Clamp style catheter connector from the Contiplex Tuohy $®$ Continuous Nerve Block Set (Fig. 1) to see if we could get a tight seal with the catheter.

None of these connectors produced a tight seal. We used a blunt tip syringe filler device inserted into the catheter to check catheter patency and to aspirate CSF. We then modified this technique by placing 2 silk sutures around the catheter segment over the syringe 


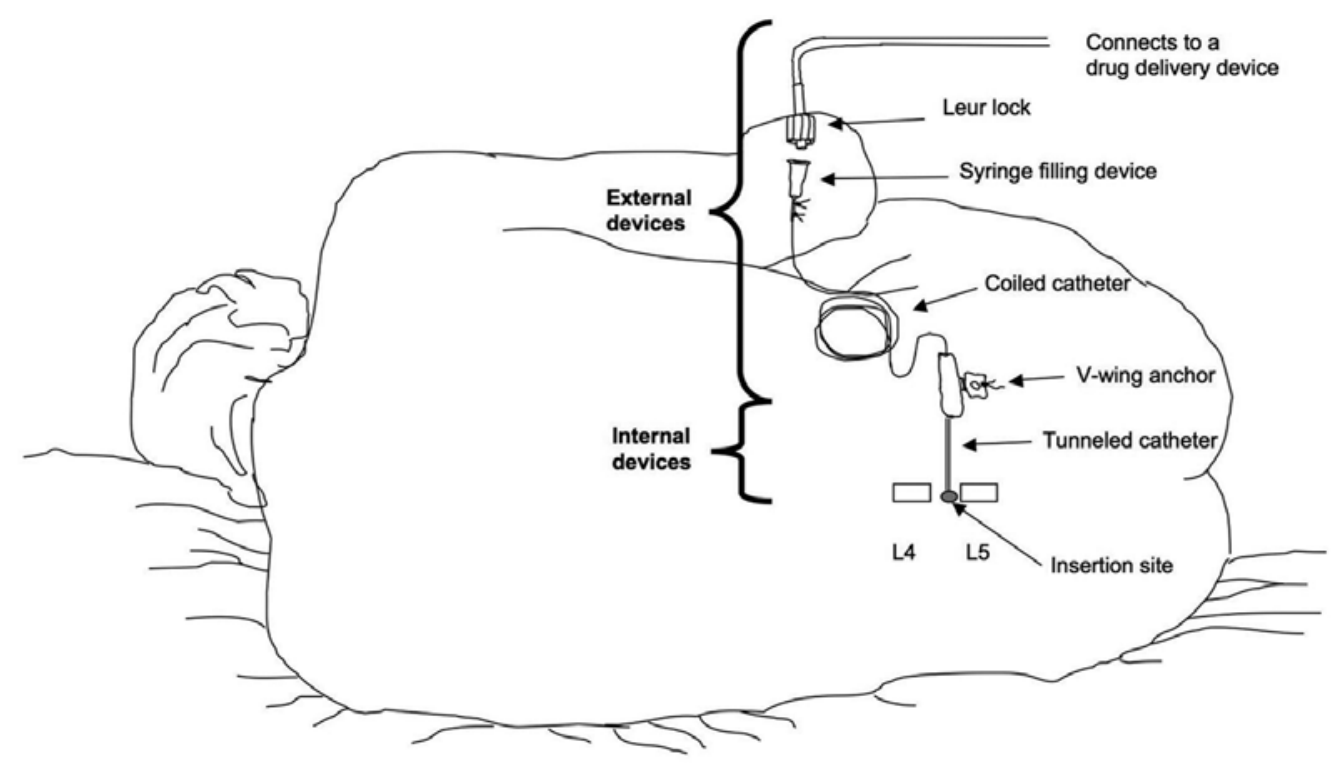

Fig. 3. Internal and external components of the intrathecal catheter.

tip. This produced a tight seal. Once the sutures are in place, the syringe tip device is difficult to dislodge or remove. With excessive force, the catheter began to stretch before the syringe tip filling device came out. Figure 2 shows the modification in detail.

As explained earlier, we placed the catheter with the patient in lateral decubitus position. The catheter was tunneled from medial to a lateral position and secured to the skin. Fig. 3 shows the internal and external components of the intrathecal catheter. The patient was comfortable and pain control was maintained in the home hospice setting for 3 weeks.

\section{Conclusion}

The Medtronic Indura 1P catheter was placed intrathecally, tunneled subcutaneously, and connected to an external drug infusion pump for a 65-year-old female with stage IV squamous cell carcinoma of the cervix with a life expectancy of 4-6 weeks. The modification allowed the use of an external pump with an intrathecal catheter for a duration of 3 weeks without significant complications. The intrathecal route of analgesia significantly reduced the patient's pain. Due to the nature of a case report, we cannot make generalized statements regarding the rates of complications, the limits of the duration of use, or the cost/benefit over other epidural catheters. Larger studies will need to be done to answer these questions.

\section{References}

1. Hassenbusch SJ, Paice JA, Patt RB, Bedder MD, Bell GK. Clinical realities and economic considerations: Economics of intrathecal therapy. J Pain Symp. Manag 1997; 14:S36-48.

2. Kedlaya D, Reynolds L, Waldman S. Epidural and intrathecal analgesia for cancer pain. Best Practice \& Research 2002; 16:651-665.
3. Bedder MD, Burchiel K, Larson A. Cost analysis of two implantable narcotic delivery systems. Journal of pain and symptom management. J Pain Symp Manag 1991; 6:368-373.

4. Patel VB, Manchikanti L, Singh V, Schultz DM, Hayek SM, Smith HS. Systematic review of intrathecal infusion systems for long-term management of chronic non-cancer pain. Pain Physician 2009; 12:345-360.

5. Manchikanti L, Boswell MV, Datta S, Fellows B, Abdi S, Singh V, Benyamin RM, Falco FJ, Helm S, Hayek SM, Smith HS; ASIPP. Comprehensive review of therapeutic interventions in manag- 
ing chronic spinal pain. Pain Physician 2009; 12:E123-E198.

6. Manchikanti L, Boswell MV, Singh V, Benyamin RM, Fellows B, Abdi S, Buenaventura RM, Conn A, Datta S, Derby R, Falco FJE, Erhart S, Diwan S, Hayek SM, Helm S, Parr AT, Schultz DM, Smith HS, Wolfer LR, Hirsch JA. Comprehensive evidence-based guidelines for interventional techniques in the management of chronic spinal pain. Pain Physi- cian 2009: 12:699-802.

7. Aprili D, Bandschapp O, Rochlitz C, Urwyler A, Ruppen W. Serious complications associated with external intrathecal catheters used in cancer pain patients: A systematic review and meta-analysis. Anesthesiology 2009; 111:1346-1355.

8. Crul BJ, Delhaas EM. Technical complications during long-term subarachnoid or epidural administration of morphine in terminally ill cancer patients: A review of 140 cases. Reg Anesth 1991; 16:209-213.

9. Burton AW, Hassenbusch SJ. The double-catheter technique for intrathecal medication trial: A brief technical note and report of five cases. Pain Medicine 2001; 2:352-354. 